\title{
Lung Nodule Detection and Analysis using VDE Chest Radiographs
}

\author{
Anoop C S \\ M.Tech Graduate \\ SCT College of Engineering \\ Thiruvananthapuram, Kerala, India
}

\author{
Preeja V \\ Assistant Professor \\ SCT College of Engineering \\ Thiruvananthapuram, Kerala, India
}

\begin{abstract}
Lung cancer becomes very common in our environment. Many computer-aided detection (CADe) schemes are available to detect lung nodules. So as to detect nodules in cost effective way, system uses chest radiographs (CXRs). Major challenge in those systems are the anatomical structures (ribs and clavicles) in the CXRs. These structures will conceal the nodules behind it. In order to overcome this problem virtual dual energy (VDE) technique has been implemented, which produces ribs and clavicle suppressed CXR image. After detecting the nodules, analysis of different types of nodules will assist the radiologist to improve the diagnosis accuracy, sensitivity and further treatment procedures. Currently the CADe scheme combine with VDE technique is having $85 \%$ sensitivity with 5 FPs/image.
\end{abstract}

\section{Keywords}

Lung nodule; CADe scheme; VDE technique.

\section{INTRODUCTION}

Nowadays, Lung Cancer became a very common disease. Lung cancer is characterized by cell growth in tissues of the lung. Early detection of the lung cancer will reduce the death rate in high propositions. Cure of lung cancer and prognosis depends on the early detection of lung nodules, which leads to cancer. A lung nodule is a spot on the lungs which is $3 \mathrm{~cm}$ in diameter or less. If it is larger than $3 \mathrm{~cm}$, it is considered as lung mass. Lung cancer patient's survival rate is only 14\% [1]. This can be easily raised to $40 \%$ if it can be detected as early as possible. Roughly half of smokers over the age of 50 will have nodules on their chest.

Computer tomography is a more sensitive imaging modality for early stage detection of lung cancer [2]. But these are not cost-effective and not routinely available. So chest radiographs are far more commonly used for chest diseases [3]. Lung nodules are missed up to $30 \%$ by radiologists in CXRs. Because those nodules were obscured by the overlying bones and clavicles [4]. It has been overcomes through soft-tissue images obtained through the Virtual Dual Energy technique.

\section{RELATED WORKS}

Computer-aided diagnosis (CAD) is a very effective approach for improving diagnostic accuracy. Many CADe schemes for nodule detection has been investigated for assisting radiologist [5]. Earlier CADe schemes still produces a relatively large number of false positives (FPs). This would reduce the efficiency of radiologists'. An observer study conducted by Matsumoto et al. showed that CADe scheme had a high FP rate of 11 per image [6]. Therefore, for a CADe scheme a low FP rate is critical. Based on the symmetry between the left and right lung regions a method called local contralateral subtraction has been proposed by Yoshida et al. to remove the anatomic structures [7]. A multiple massive- training artificial neural networks (MTANNs) employed to reduce the number of FPs produced by CADe scheme had developed by the Suzuki et al [8]. Major challenge was to detect nodules behind the anatomic structures [9]. For separating soft tissue from bones dual-energy subtraction technique [10] can be used by the use of two X-ray exposures at two different energy levels. But, a limited number of hospitals use this system, because specialized equipment are required, and the radiation dose can be double. Suzuki et al. developed an image-processing technique called virtual dual energy (VDE) radiography for suppressing ribs and clavicles in CXRs by means of a multi-resolution MTANN.

In this work, a CADe scheme for detection and analysis of pulmonary nodules by use of the MTANN VDE technology has been developed to assist radiologists in improving their sensitivity by reducing the FP rate.

\section{METHODOLGY}

In this CADe scheme there are two main phases. The first phase is the detection of nodules which has four major steps: A) segmentation of lung region based on multi-segment active shape model (M-ASM); B) two stage nodule enhancement techniques; C) segmentation of nodule candidates using K-Mean Clustering algorithm; and finally D) feature analysis and classification of the nodule candidates into nodules or non-nodules. And the second phase consist of the analysis of the detected nodules.

\subsection{Segmentation of lung region}

Segmentation of lung region has done based on the MASM technique, which is the improved model of the Cootes et al. formulation [11] by specifying the structural boundaries called transitional landmarks. Multiple segments such as the heart, aorta, and rib-cage will determines transitional landmarks. Thus the transitional landmarks perform likes feature nodes. This allowed the nodes to learn the boundary type of each local appearance models, rather than generalizing. This improves the boundary accuracy. The figure 1 shows the transitional landmarks across multiple segments [12].

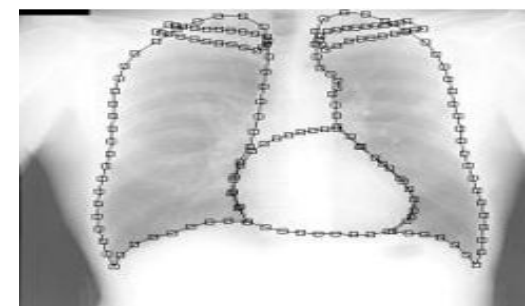

Figure 1: Transitional landmarks. 


\subsection{Nodule Enhancement Techniques}

This two-stage nodule enhancement technique produced an image with enhanced nodules and a nodule likelihood map. In the first step, the technique enhances nodules by use of gray-level morphologic opening operators. Figure 2 shows the first step [13].

In the second step the nodule likelihood map has been generated from the nodule enhanced image by use of the directional gradient magnitude filter. Figure 3 shows the local peaks in the likelihood map.

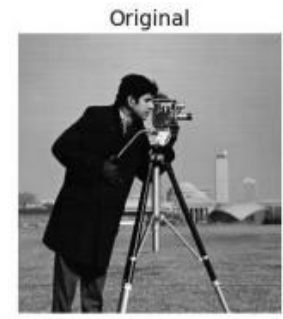

Gray-level opening

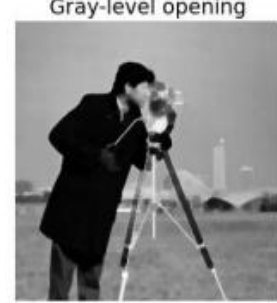

Gray-level closing

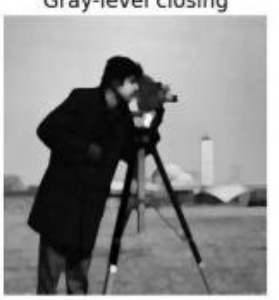

Morphological gradient

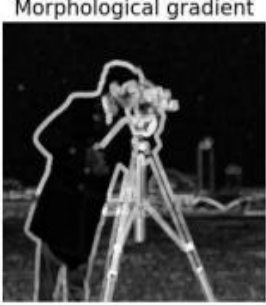

Figure 2: Enhancement techniques.

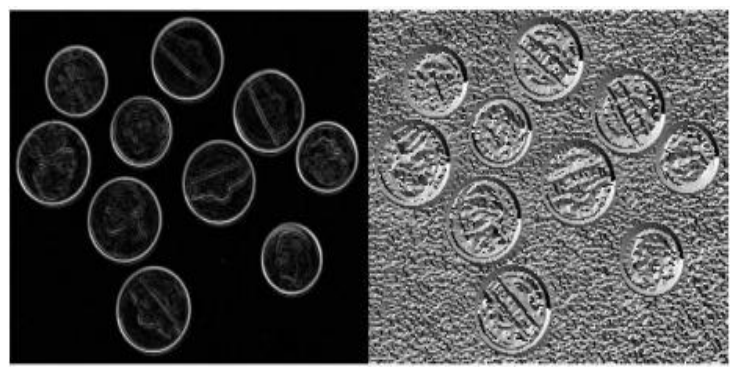

Figure 3: Directional gradient magnitude filter.

\subsection{Segmentation of nodule candidates}

Morphologic filtering and K-mean clustering techniques are used in order to segment the nodule candidates. In morphologic filtering erosion and dilation operators are implemented to break the connection between the nodule and non-nodule candidates and to dilate the connected regions respectively. Filtering resulted in a rough nodule candidate region. To segment the candidate K-mean clustering has been used. Peaks in the enhanced image used as initial parameter for the algorithm.

In clustering method, first, a primary cluster was defined that contains the nodule candidate location. Next, clusters connected to primary clusters were added. The connected clusters were identified by the minimum value between the peaks and each of the other peaks was larger than a threshold value.

\subsection{Feature Analysis and Classification}

Segmentation resulted in nodule candidates. Features of those candidates were extracted. Features include shape, gray-level, texture, etc. Feature extraction is used to detect the odd ones.

\section{VDE IMAGE CREATION}

Multi-resolution MTANN leads to the development of an image processing technique for the suppression of anatomic structures in CXRs. This technique resulted in white soft tissues such as lung nodules and vessels. Figure 4 shows the Original CXR and its VDE image [14]. Bone images or teaching images are obtained by use of dual energy radiography systems.
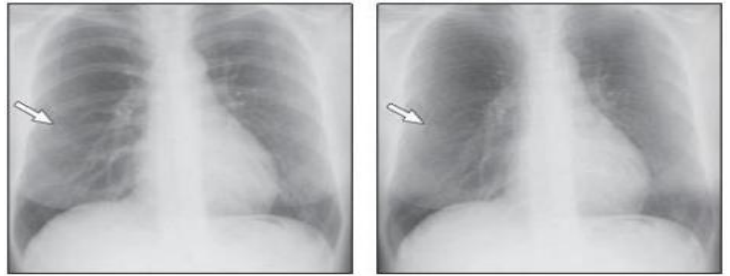

Figure 4: Original CXR and its VDE image. Arrows indicate the nodule region.

Ribs and clavicle suppression using MTANN model on CXRs involves: a training step and an application step. Figure 5 indicates the working of MTANN [15]. In training step, dual-energy subtraction technique used for separating bone from soft tissue on CXRs, produces two tissue-selective images, that is, a bone image and a soft tissue image. Original CXRs are used as input images to the MTANN, and the corresponding bone images are used as the teaching images. In application step, an input is given to the trained MTANN, it output a bone image on which the bones were isolated and enhanced. A soft tissue image on which ribs were suppressed was produced by subtraction of the bone image from the input chest image.

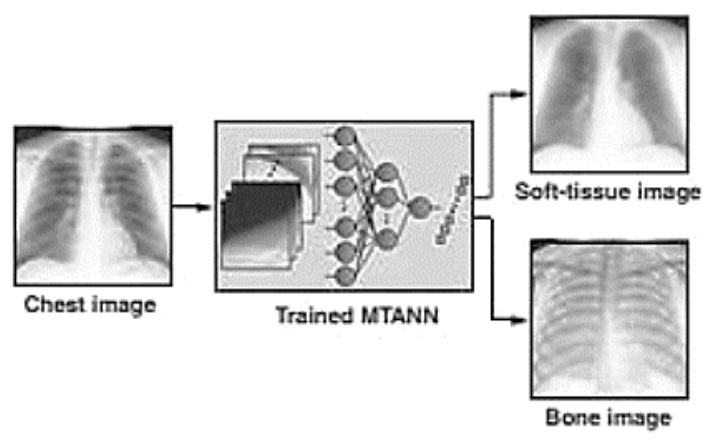

Figure 5 MTANN Process.

The suppression of ribs containing various frequencies was difficult for a single MTANN. Multi-resolution decomposition/composition techniques were employed to overcome the issue. Figure 6 specifies the execution flow of MTANN [16]. Initially, a lower resolution image was obtained by performing down sampling with averaging on an original higher resolution image. Then up-sampling with pixel substitution has been employed to enlarge lower resolution image. After that, subtraction of the enlarged lower resolution image from the higher resolution image will result in a higher resolution difference image. Same procedures are performed repeatedly. Thus, multi-resolution images having various frequencies are obtained by use of the multi-resolution decomposition technique. 


\section{CADE SCHEME WITH VDE TECHNIQUE}

Here both the original CXRs and VDE CXRs taken as inputs. In order to reduce the false positives VDE CXRs were used. Most of the FP nodules were located in the rib crossing, clavicles regions in the original CADe scheme. Some nodules have features like bones in terms of shape, size, contrast, and orientation. These nodules may be suppressed in CADe scheme with VDE technique. The figure 7 describes the proposed system [1].
The second phase in this scheme describes the analysis of the nodule candidates. This phase will assist the radiologist in order to improve the sensitivity and accuracy in detecting the pulmonary nodules using the low cost CXRs. Its efficiency results in one of the most cost effective technique as compared to other techniques. Through analysis it can be find out that whether the patients having any type of nodules like calcification, histoplasmoma, popcorn calcification, hamartoma, and rheumatoid nodules. These nodules were analysed based on the features like shape, size, gray- level and its orientation.

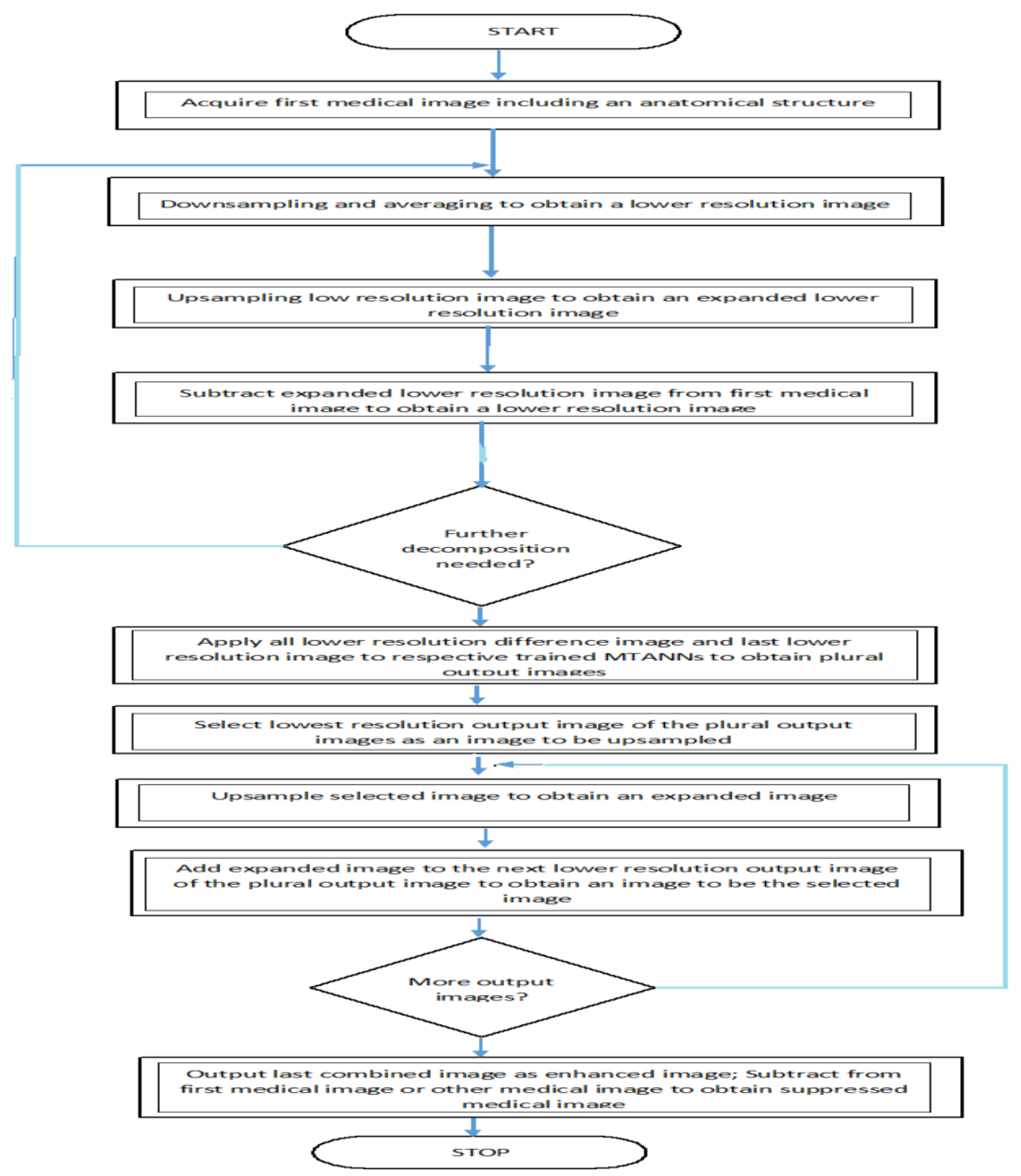

Figure 6: MTANN process flow.

Each of the nodules differs with their features and makes easier for this scheme to identify the type of nodule. Also, the patient's treatment stage and treatment details can be predicted with analysis phase. 


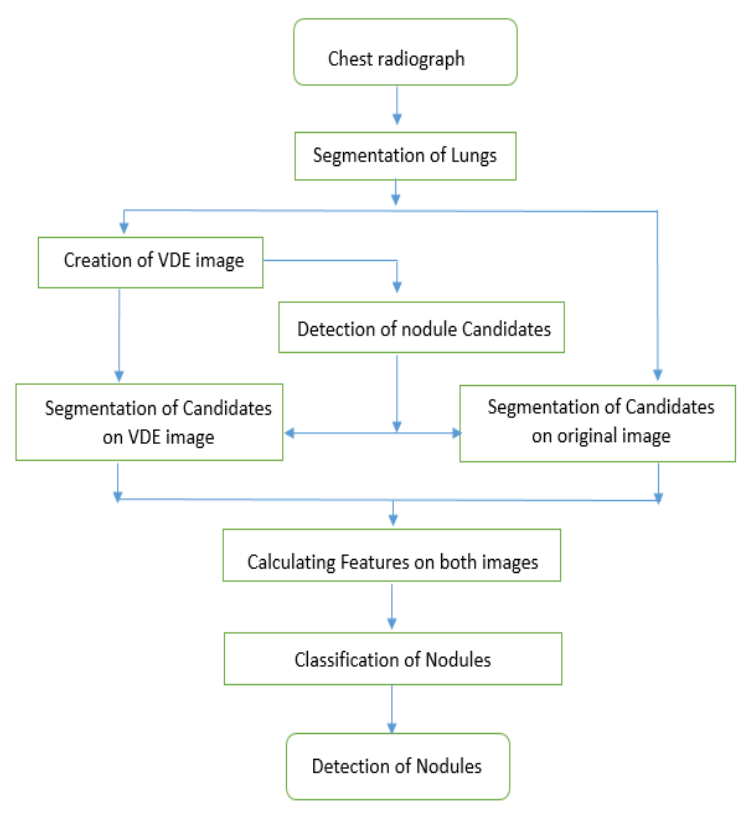

Figure 7 Flow Diagram

\section{EXPERIMENTAL RESULTS}

The experimental results shows the VDE technique efficiency and the FP rate reduction in nodule detection as compared to the original CADe scheme. Figure 8 shows the change in rib contrast to create the soft tissue images. (a) original image, (b) $20 \%$ rib contrast, (c) $40 \%$, (d) $70 \%$, (e) $90 \%$, and (f) bone image [1].

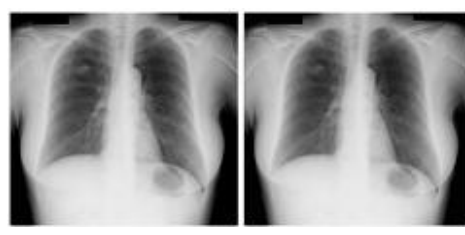

(a)

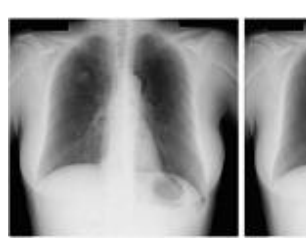

(d) (b)

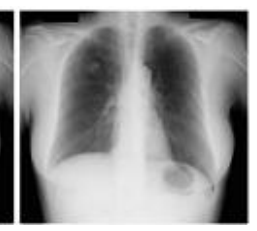

(c)

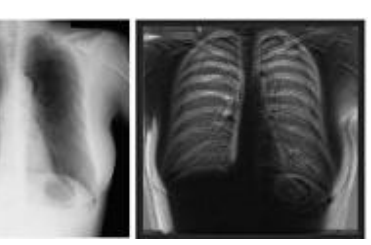

(e) (f)
Figure 8 Illustration of change in rib contrast.

\subsection{MTANN Training}

The training samples were extracted manually in order to cover the samples with nodules and non-nodule images. Figure 9 specifies the operating characteristic curves of diagnostic performance done by the radiologists. Table I following it shows the area under the best fit receiver operating characteristic (ROC) curve (AUC) analysis by certified radiologists [15].

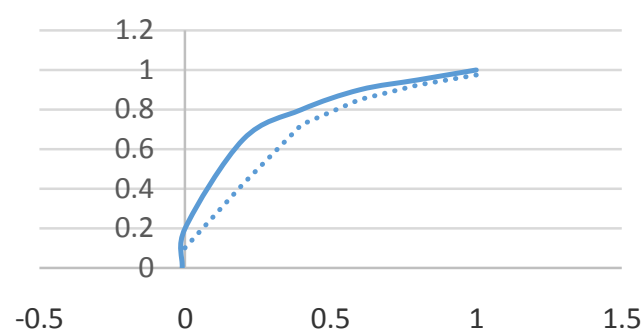

Figure 9: Graph shows the diagnostic performance of radiologist reading the images with and without rib suppression.

Table I: AUC Analysis.

\begin{tabular}{|l|l|l|}
\hline \multirow{2}{*}{ Observer } & \multicolumn{2}{|c|}{ Area Under Curve } \\
\cline { 2 - 3 } & $\begin{array}{l}\text { Without rib } \\
\text { suppression }\end{array}$ & $\begin{array}{l}\text { With rib } \\
\text { suppression }\end{array}$ \\
\hline \multicolumn{2}{|l|}{ Certified radiologists } \\
\hline 1 & 0.859 & 0.962 \\
\hline 2 & 0.936 & 0.880 \\
\hline 3 & 0.856 & 0.910 \\
\hline 4 & 0.896 & 0.821 \\
\hline 5 & 0.773 & 0.820 \\
\hline 6 & 0.841 & 0.913 \\
\hline 7 & 0.775 & 0.890 \\
\hline Mean \pm SD & $0.816 \pm 0.077$ & $0.843 \pm 0.074$ \\
\hline 6.2 Nodul
\end{tabular}

6.2 Nodule Candidate Detection

The true positive (TP) rate improved by adopting the VDE images. Earlier the bone image was subtracted from the original CXR to get the soft tissue image. From that process the sensitivity was highest when rib contrast parameter set to 0.4 . Figure 10 shows the performance of nodule detection stage with different rib contrast [1].

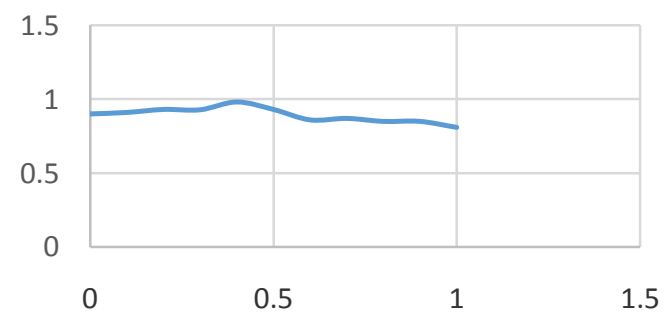

Figure 10: Effect of change in rib contrast on sensitivity.

Performance of the CADe scheme using VDE images only was higher than that of the original scheme. It achieved sensitivity of $65.7 \%$ and $60.7 \%$ for original scheme at a FP rate of 1.5 FPs per image [1].

Performance of the CADe scheme when combining both VDE technique with original scheme achieved a sensitivity of $85.0 \%$ at FP rate of 5 FPs per image. Figure 12 shows the improvement in nodule detection with both 
schemes: (a) False negatives (arrow) of original CADe scheme. (b) TPs (arrow) of VDE based CADe scheme [1].

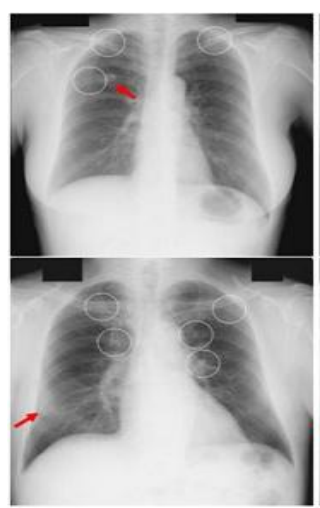

(a)

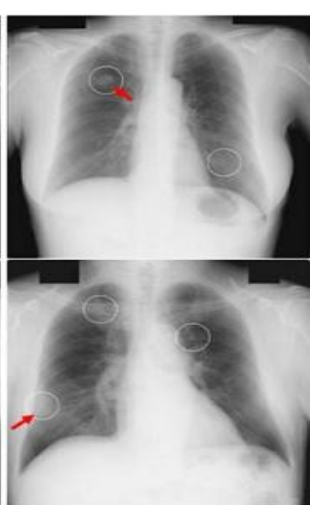

(b)
Figure 12: Illustration of the improvement in nodule detection.

\subsection{Performance Comparison}

Definitive comparison with other CADe schemes were difficult because of different databases, different evaluation TP criteria, different evaluation procedures, and different operating points [17]. Table II specifies the sensitivity of different schemes [1].

Table II: Performance Comparison.

\begin{tabular}{|l|l|l|}
\hline & $\begin{array}{l}\text { Sensiti } \\
\text { vity }\end{array}$ & FPs/image \\
\hline Wei et al. (2002) [18] & $80 \%$ & 5.4 \\
\hline $\begin{array}{l}\text { Coopini et al. (2003) } \\
{[19]}\end{array}$ & $60 \%$ & 4.3 \\
\hline $\begin{array}{l}\text { Schiham et al. (2006) } \\
{[14]}\end{array}$ & $67 \%$ & 4.0 \\
\hline Hardie et ai. (2009) [20] & $80 \%$ & 5.0 \\
\hline Chen et al. (2011) [21] & $79 \%$ & 5.0 \\
\hline VDE-based CADe & $85 \%$ & 5.0 \\
\hline
\end{tabular}

\section{CONCLUSION}

In this work a supervised CAD scheme with VDE technique has been proposed. This will assist the radiologist to improve the accuracy and sensitivity. Through analysis different types of nodules can be specified and proper treatment can be mentioned by the radiologist for patients. As future enhancement the performance of this system (85\% sensitivity with 5 FPs/image) can be improved and can be used as a primary cancer detection tool at lower cost.

\section{REFERENCES}

[1] Sheng Chen, and Kenji Suzuki, "Computerized Detection of Lung Nodules by Means of "Virtual Dual-Energy" Radiography", IEEE TRANSACTIONS ON BIOMEDICAL ENGINEERING, VOL. 60, NO. 2, FEB 2013.
[2] C. I. Henschke, D. I. McCauley, D. F. Yankelevitz, D. P. Naidich, G. McGuinness, O. S. Miettinen, D. M. Libby, M. W. Pasmantier, J. Koizumi, N. K. Altorki, and J. P. Smith, "Early lung cancer action project: Overall design and findings from baseline screening,'Lancet, vol. 354, pp. 99-105, Jul. 10, 1999.

[3] G. P. Murphy, W. Lawrence, R. E. Lenhard, and American Cancer Society,American Cancer Society Textbook of Clinical Oncology, 2nd ed. Atlanta, GA.: American Cancer Society, 1995.

[4] J. H. Austin, B. M. Romney, and L. S. Goldsmith, "Missed bronchogenic carcinoma: Radiographic findings in 27 patients with a potentially resectable lesion evident in retrospect,"Radiology, vol. 182, pp. 115-122, Jan. 1992.

[5] M. L. Giger, K. Doi, and H. MacMahon, "Image feature analysis and computer-aided diagnosis in digital radiography. 3. Automated detection of nodules in peripheral lung fields,"Med. Phys., vol. 15, pp. 158-166, Mar.-Apr. 1988.

[6] T. Matsumoto, K. Doi, A. Kano, H. Nakamura, and T. Nakanishi, "Evaluation of the potential benefit of computer-aided diagnosis (CAD) for lung cancer screenings using photofluorography: Analysis of an observer study,"Nippon Igaku Hoshasen Gakkai Zasshi, vol. 53, pp. 1195-1207, Oct. 25, 1993.

[7] H. Yoshida, "Local contralateral subtraction based on bilateral symmetry of lung for reduction of false positives in computerized detection of pulmonary nodules,'IEEE Trans. Biomed. Eng., vol. 51, no. 5, pp. 778-789, May 2004.

[8] K. Suzuki, J. Shiraishi, H. Abe, H. MacMahon, and K. Doi, "False-positive reduction in computer-aided diagnostic scheme for detecting nodules in chest radiographs by means of massive training artificial neural network," Acad. Radiol., vol. 12, pp. 191201, Feb. 2005.

[9] T. Matsumoto, H. Yoshimura, K. Doi, M. L. Giger, A. Kano, H. MacMahon, K. Abe, and S. M. Montner, "Image feature analysis of false-positive diagnoses produced by automated detection of lung nodules,"Investigative Radiol., vol. 27, pp. 587597, Aug. 1992.

[10] S. Kido, K. Kuriyama, C. Kuroda, H. Nakamura, W. Ito, K. Shimura, and H. Kato, "Detection of simulated pulmonary nodules by single-exposure dual-energy computed radiography of the chest: Effect of a computeraided diagnosis system (Part 2), ”Eur. J. Radiol., vol. 44, pp. 205-209, Dec. 2002.

[11] T. F. Cootes, A. Hill, C. J. Taylor, and J. Haslam, "Use of active shape models for locating structures in medical images,'Image Vis. Comput., vol. 12, pp. 355-365, 1994.

[12] Bram van Ginneken, Mikkel B. Stegmann, and Marco Loog, "Segmentation of anatomical structures in chest, radiographs using supervised methods: a comparative study on a public database Revised version”, Aug-2004.

[13] J. Serra, Image Analysis and Mathematical Morphology,vol.1. New York: Academic, 1982. 
[14] A. M. Schilham, B. van Ginneken, and M. Loog, "A computer-aided diagnosis system for detection of lung nodules in chest radiographs with an evaluation on a public database,"Med. Image Anal., vol. 10, pp. 247-258, Apr. 2006.

[15] Seitaro Oda, Kazuo Awai, Kenji Suzuki, Yumi Yanaga, Yoshinori Funama, Heber MacMahon, Yasuyuki Yamashita, "Performance of Radiologists in Detection of Small Pulmonary Nodules on Chest Radiographs: Effect of Rib Suppression With a Massive-Training Artificial Neural Network ", AJR2009; 193:W397-W402 0361-803X/09/1935W397, NOV 2009.

[16] Kenji Suzuki et al, Kunio Doi et al, "Image Modification and detection using Massive training artificial neural network (MTANN)", The University of Chicago, Jun-2009.

[17] R. M. Nishikawa, M. L. Giger, K. Doi, C. E. Metz, F. F. Yin, C. J. Vyborny, and R. A. Schmidt, "Effect of case selection on the performance of computeraided detection schemes," Med. Phys., vol. 21, pp. 265-269, Feb. 1994.
[18] J. Wei, Y. Hagihara, A. Shimizu, and H. Kobatake, "Optimal image feature set for detecting lung nodules on chest X-ray images," in Proc. Comput.Assisted Radiol. Surg., 2002, pp. 706-711.

[19] G. Coppini, S. Diciotti, M. Falchini, N. Villari, and G. Valli, "Neural networks for computer-aided diagnosis: Detection of lung nodules in chest radiograms,"IEEE Trans. Inf. Technol. Biomed., vol. 7, no. 4, pp. 344-357, Dec. 2003.

[20] R. C. Hardie, S. K. Rogers, T. Wilson, and A. Rogers, "Performance analysis of a new computer aided detection system for identifying lung nodules on chest radiographs,"Med. Image Anal., vol. 12, pp. 240-258, Jun. 2008.

[21] S. Chen, K. Suzuki, and H. MacMahon, "Development and evaluation of a computer-aided diagnostic scheme for lung nodule detection in chest radiographs by means of two-stage nodule enhancement with support vector classification," Med. Phys., vol. 38, pp. 1844-1858, 2011. 